\title{
Evaluación de un curso itinerante sobre aprendizaje basado en problemas a través de dos encuestas
}

\author{
Laura García-Martín, Elisabet Serés, Rosa M. Torrens, Luis A. Branda, Fèlix Bosch
}

Introducción. En un intento por promover la formación en aprendizaje basado en problemas (ABP) entre diferentes profesionales de la docencia, la Fundación Dr. Antonio Esteve decidió organizar varios cursos en diversas ciudades españolas en colaboración con otras instituciones. Este estudio presenta la experiencia de cuatro años de formación en ABP (20082011) promovidos desde esta fundación.

Sujetos y métodos. Se analizaron los datos de 11 cursos sobre ABP a los que asistieron 216 profesionales. En primer lugar, se obtuvo información sobre la satisfacción de los asistentes a partir de un primer cuestionario impreso entregado al final de cada curso. En segundo lugar, se analizaron datos adicionales obtenidos a partir de un segundo cuestionario de opinión enviado por correo electrónico meses después de finalizar el curso.

Resultados. La satisfacción con el curso de los 194 participantes que completaron el primer cuestionario (tasa de respuesta del 90\%) fue muy alta (mediana de 4 sobre 5). Un total de 54 participantes (tasa de respuesta del 25\%) respondieron al segundo cuestionario, según el cual los participantes mejoraron sus conocimientos, actitudes y habilidades con una mediana de incremento de 3 para la mayoría de parámetros evaluados. Después del curso, el 39\% de los asistentes indicaron que pudieron implementar el ABP en alguna de sus actividades docentes.

Conclusiones. Los cursos fueron bien acogidos y han contribuido al aprendizaje en general por parte de los asistentes. Los cuestionarios también han servido para poner de relieve algunos de los problemas que limitan la aplicación del ABP en nuestro entorno.

Palabras clave. ABP. Aprendizaje autodirigido. Aprendizaje basado en problemas. Cuestionario. Docencia. Educación médica. Evaluación de programas. Formación de posgrado.

\section{Survey-based assessment of a workshop conducted in several Spanish cities on problem-based learning}

Introduction. In an attempt to promote problem-based learning (PBL), the Esteve Foundation organized several seminars for various teaching professionals in a number of Spanish cities. This study presents the experience gained in four years of running these courses (2008-2011), which were offered in collaboration with other institutions.

Subjects and methods. Data were analyzed from 11 PBL courses involving 216 professionals. Firstly, satisfaction data were taken from a printed survey conducted at the end of each course. Secondly, additional data were obtained from a deferred opinion questionnaire sent by email several months after the course.

Results. Satisfaction data for 194 attendees who completed the first questionnaire (response rate of 90\%) were very positive (median: 4 out of 5). Only 54 attendees (response rate of $25 \%$ ) responded to the deferred questionnaire. Participants improved their knowledge, attitude, and skills (median increase: 3 out of 5) in most of the parameters evaluated. Furthermore, after completion of the course, 39\% of the attendees reported that they were able to implement PBL in some of their teaching activities.

Conclusions. The courses were well received and contributed to the overall learning of the attendees. The questionnaires served to highlight some of the major problems in the implementation of PBL.

Key words. Medical education. PBL. Postgraduate training. Problem-based learning. Programme evaluation. Questionnaire. Self-directed learning. Undergraduate teaching.
McMaster University; Canadá (L.A. Branda). Fundación Dr. Antonio Esteve (L. García-Martín, E. Serés, F. Bosch). Exdirectora Escuela Universitaria de Enfermería; Hospital Vall d'Hebron (R.M. Torrens). Departamento de Ciencias Experimentales y de la Salud: Universitat Pompeu Fabra; Barcelona, España (F. Bosch).

Correspondencia:

Dr. Fèlix Bosch. Fundación Dr. Antonio Esteve. Llobet i Vall-Llosera, 2. E-08032 Barcelona.

E-mail:

fbosch@esteve.org

Agradecimientos:

Los autores quieren agradecer a todos los participantes de los cursos de formación sobre ABP por haber confiado en los organizadores $y$ profesores responsables. En especial, se quiere destacar la amabilidad de aquellos que aceptaron responder los cuestionarios de evaluación. Puesto que estas actividades formativas no hubieran tenido lugar sin su inestimable ayuda y contribución, los autores desean agradecer la labor de E. Caballería y P. Morales, ambos de la Fundación Dr. Antonio Esteve, y de los representantes de cada una de las instituciones colaboradoras con los cursos de formación en ABP: C. Altés y M. Portugalete (Colegio Oficial de Biólogos de Euskadi, Bilbao); R. Brugada y M. Aymerich (Facultad de Medicina, Universitat de Girona); C. García Cruz y S. Sintes (Escuela de Verano de Salud Pública, Maó); P. d’Ocón y M.D. Ivorra (Facultad de Farmacia, Universitat de València); M.A. Fontenla (Facultad de Farmacia, Universidad de Santiago de Compostela); M.R. Olóriz y M.A. Marquínez (Hospital Universitario Marqués de Valdecilla, Santander); J. Gálvez y S. Risco

(Facultad de Farmacia, Universidad de Granada); J.J. Rodrigo, P. Rodríguez y M.A. Saavedra (Hospital Universitario de Gran Canaria Dr. Negrín, Las Palmas); 
y E. Serratusell y N. Sansó (Grupo de Gestión Sanitaria de Mallorca, Palma de Mallorca)

Datos preliminares de este artículo se presentaron como póster en el 6th European Congress of Pharmacology; Granada, 17-20 de julio de 2012

Conflicto de intereses: No declarado.

Competing interests: None declared.

(C) 2014 FEM

\section{Introducción}

El aprendizaje basado en problemas (ABP) es una estrategia coherente con las directivas del Espacio Europeo de Educación Superior y el planteamiento de la Declaración de Bolonia [1]. Básicamente, el ABP se fundamenta en un proceso de enseñanza y aprendizaje que pretende facilitar el desarrollo de competencias que deberían permitir a los estudiantes desarrollar una gestión más autónoma de su aprendizaje. Este proceso pone énfasis en un aprendizaje autodirigido donde el estudiante es el protagonista y no un mero receptor pasivo de la enseñanza impartida por el profesor [2].

Durante los últimos años, en España se han puesto en marcha diferentes iniciativas docentes mediante el empleo del ABP a fin de incorporar nuevos métodos a la docencia universitaria tradicional. En algunos centros se ha optado por desarrollar proyectos integrales de ABP [3,4], mientras que en otros se ha considerado más adecuado seguir modelos mixtos donde se combina el ABP con otros formatos docentes $[1,5]$. Si bien estas y otras aproximaciones pueden suponer importantes avances en la docencia universitaria, no siempre están exentas de riesgo [6]. Con riesgo o sin él, la situación actual no puede considerarse del todo satisfactoria puesto que desde el entorno académico universitario todavía se demanda formación en nuevas estrategias docentes entre las que se incluye el ABP [7].

Siguiendo la línea de seminarios de formación anteriores realizados en diferentes áreas de ciencias de la salud que organiza la Fundación Dr. Antonio Esteve [8,9], a finales de 2007 se diseñó un programa formativo a fin de contribuir a divulgar las bases del ABP. El objetivo final de este curso era dar a conocer la metodología docente del ABP entre profesores universitarios y otros docentes interesados. Estos cursos se desarrollaron a través de 11 ediciones que tuvieron lugar en diferentes ciudades españolas en colaboración con instituciones académicas y asistenciales.

Si bien la mayoría de las universidades españolas llevan a cabo programas de formación de distinta magnitud, los resultados de su impacto no se han explorado de forma extensiva. Por ello, el presente artículo pretende investigar los logros y respuestas de los participantes a través de la experiencia de formación en ABP desde la Fundación Dr. Antonio Esteve durante cuatro años (2008-2011). El análisis se fundamenta en los resultados obtenidos mediante dos cuestionarios de evaluación sobre la satisfacción con los cursos de formación y la opinión de los asistentes tras la realización de dichos cursos. Entre otros aspectos, se evalúa la aceptación del curso y el grado de mejora en los conocimientos, habilidades y actitudes según la opinión de los participantes. También se interrogó sobre la posibilidad de aplicación del ABP en su entorno docente y profesional.

\section{Sujetos y métodos}

\section{Características de los cursos de formación}

Entre 2008 y 2011 se organizaron 11 ediciones de cursos de formación sobre ABP con un formato y contenidos muy similares. Todas ellas las impartieron dos de los autores del presente artículo (R.M.T. y L.A.B.) y se organizaron desde la Fundación Dr. Antonio Esteve en colaboración con otras instituciones académicas de diferentes ciudades españolas (véanse en 'agradecimientos'). La tabla I resume las principales características de estas ediciones que reunieron a un total de 216 participantes.

Para la realización de estos cursos, previamente se publicitó la información a través de la Fundación Dr. Antonio Esteve y de las instituciones colaboradoras, a partir de sus propias listas de correo electrónico y páginas de Internet, así como por correo postal mediante los correspondientes trípticos de divulgación. El precio predefinido de la inscripción a los cursos era de $100 €$ por persona, acordándose subvenciones especiales con las diferentes instituciones académicas colaboradoras. Se pactó no exceder los 25 participantes en cada edición del curso. Estas actividades formativas se orientaron fundamentalmente a docentes universitarios, sin excluir la participación de otros docentes o profesionales interesados en mejorar sus competencias sobre el ABP.

La duración de cada edición fue de dos días consecutivos en formato intensivo con sesiones de mañana y tarde (de 09:00 a 18:30 h), completándose un total de 16 horas de docencia. Los contenidos del curso (Tabla II) se impartían en formato de sesión plenaria o en grupos siguiendo la propia metodología del ABP. Los dos profesores del curso también impartieron clases magistrales. Dichos contenidos se modificaron ligeramente durante las diferentes ediciones del curso, atendiendo las opiniones de los asistentes de sesiones previas y en función de una mejor adecuación a las necesidades de los participantes. Como parte de la documentación del curso, cada asistente recibió el programa con los horarios de los dos días, bibliografía general, documentos de apoyo y un cuaderno sobre el ABP publicado por la Fundación Dr. Antonio Esteve [10]. 


\section{Cuestionario 1 inmediato de satisfacción}

Al finalizar cada edición del curso, se distribuyó un formulario impreso (cuestionario 1) entre todos los asistentes a fin de evaluar de forma inmediata su satisfacción con la actividad desarrollada. La información recogida resultó también útil para mejorar la preparación y el desarrollo de futuras ediciones del curso. Este formulario incluía preguntas sobre la valoración general del curso, la adecuación de la documentación entregada, el contenido y duración de la actividad formativa, así como la valoración de los asistentes sobre el profesorado y la organización. Cada parámetro se puntuó mediante una escala tipo Likert entre 0 (completamente inadecuado) y 5 (muy adecuado). Se obtuvo la opinión de un total de 194 personas, es decir, el 89,8\% de los 216 asistentes a los cursos de formación.

\section{Cuestionario 2 diferido de opinión}

Tras cuatro años de actividad formativa a través de 11 ediciones del curso, se preparó un cuestionario (cuestionario 2) para evaluar, según la opinión de los asistentes, la utilidad y posibilidad de aplicación de este tipo de cursos de formación sobre el ABP. Se preparó una carta de presentación y justificación del cuestionario que constituía el mensaje del correo electrónico enviado a todos los asistentes de los cursos invitándolos a que respondieran las preguntas del cuestionario que se adjuntaba. Las instrucciones para su cumplimentación aparecían en el mismo formulario, así como la solicitud de que lo devolvieran por correo electrónico. El formulario contenía preguntas sobre sus datos personales (edad, sexo, formación y lugar de trabajo) y si disponían de experiencia previa sobre el ABP antes de acudir al curso de formación. En otro apartado se interrogaba sobre diversos conocimientos, actitudes y habilidades antes y después del curso, que debían puntuar mediante una escala tipo Likert con valores entre 0 (mínimo valor) y 5 (máximo valor) (en la tabla III aparecen las preguntas sobre competencias del cuestionario 2). En el cuestionario 2 también se solicitaba si tras la realización del curso sobre ABP los asistentes habían podido aplicar esta metodología en su labor docente y profesional y, posteriormente, que justificasen su respuesta comentando los principales problemas que limitaban, a su entender, la implementación del $\mathrm{ABP}$ en su entorno docente. Finalmente se preguntaba su opinión sobre la necesidad de organizar cursos de formación en ABP, si recomendarían concretamente este curso recibido y, además, se dejaba un espacio abierto para comentarios y sugerencias.
Tabla I. Características generales de los cursos de formación.

\begin{tabular}{|c|c|c|c|}
\hline & Edición, ciudad & Institución organizadora a & Asistentes $^{b}$ \\
\hline \multirow{2}{*}{$\begin{array}{l}\text { Año } \\
2008\end{array}$} & 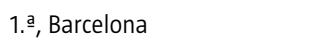 & Fundación Dr. Antonio Esteve & 12 \\
\hline & 2. ${ }^{\mathrm{a}}$, Madrid & Fundación Dr. Antonio Esteve & 16 \\
\hline \multirow{3}{*}{$\begin{array}{l}\text { Año } \\
2009\end{array}$} & 3. ${ }^{a}$, Bilbao & Colegio Oficial de Biólogos de Euskadi & 17 \\
\hline & 4. a , Girona & Facultad de Medicina, Universitat de Girona & 21 \\
\hline & 5. ^, Maó & Escuela de Salud Pública de Menorca & 22 \\
\hline \multirow{3}{*}{$\begin{array}{l}\text { Año } \\
2010\end{array}$} & 6. $\stackrel{a}{\text {, Valencia }}$ & Facultad de Farmacia, Universitat de València & 24 \\
\hline & 7. a, Santiago de Compostela & $\begin{array}{l}\text { Facultad de Farmacia, } \\
\text { Universidad de Santiago de Compostela }\end{array}$ & 24 \\
\hline & 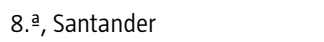 & Hospital Universitario Marqués de Valdecilla & 21 \\
\hline \multirow{3}{*}{$\begin{array}{l}\text { Año } \\
2011\end{array}$} & 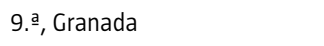 & Facultad de Farmacia, Universidad de Granada & 23 \\
\hline & 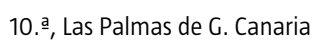 & Hospital Universitario de Gran Canaria Dr. Negrín & 17 \\
\hline & 11. a , Palma de Mallorca & Grupo de Gestión Sanitaria de Mallorca & 19 \\
\hline
\end{tabular}

a Salvo en las dos primeras ediciones del curso, que se organizaron exclusivamente desde la Fundación Dr. Antonio Esteve, el resto tuvieron lugar en colaboración con ésta y las diversas instituciones que se detallan en la tabla (en el apartado de agradecimientos se completa la información sobre los colaboradores). ${ }^{b} \mathrm{~A}$ las 11 ediciones de cursos sobre ABP asistieron un total de 216 personas, 147 mujeres $(68,1 \%)$ y 69 hombres (31,9\%). Para más información sobre el perfil de los asistentes, véase la tabla IV.

Tabla II. Principales contenidos impartidos durante los cursos

Introducción al seminario y aspectos generales sobre el ABP

Preparación de objetivos de aprendizaje

Preparación de situaciones problemáticas

Conceptos generales sobre el ABP

$\mathrm{ABP}$ en grupos grandes

ABP en grupos pequeños

Búsqueda de información relativa al plan de trabajo

Herramientas para la evaluación del estudiante

Gestión de la implementación del ABP integral en un centro docente

Sesiones de discusión general

En función de cada curso se seleccionaron los contenidos más adecuados según el criterio de los profesores y la propia elección de los asistentes durante las diferentes sesiones. Estos contenidos se impartían en formato de sesión plenaria o en grupos de trabajo (grandes o pequeños), siguiendo la propia metodología del ABP y combinado con clases magistrales. 
Tabla III. Preguntas formuladas mediante el cuestionario 2 diferido de opinión para evaluar la mejora de competencias con la realización del curso.

Antes Después

\begin{tabular}{|c|c|}
\hline & Sobre los fundamentos del ABP en general \\
\hline & Sobre el funcionamiento de una sesión de ABP en grupos pequeños \\
\hline \multirow[t]{4}{*}{ Conocimientos } & Sobre el funcionamiento de una sesión de ABP en grupos grandes \\
\hline & Sobre la evaluación del ABP \\
\hline & Sobre cómo implementar el sistema de ABP en un centro docente \\
\hline & Frente a la necesidad de emplear el ABP en docencia \\
\hline \multirow[t]{3}{*}{ Actitudes } & Respecto a la comprensión de las dificultades del ABP \\
\hline & En intentar emprender iniciativas con el ABP en mi entorno docente \\
\hline & Capacidad de preparar unos objetivos en ABP \\
\hline \multirow[t]{2}{*}{ Habilidades } & Capacidad de actuar como tutor-facilitador en una sesión de ABP \\
\hline & Capacidad para preparar el texto de un caso/situación de ABP \\
\hline
\end{tabular}

Durante el mes de agosto de 2011 se enviaron por correo electrónico 213 cuestionarios (puesto que no se disponía de la dirección electrónica de tres de los asistentes al curso). De ellos, en primera instancia, se obtuvieron 35 cuestionarios cumplimentados (16,4\%). Tras el envío de un segundo recordatorio, también por correo electrónico en octubre de 2011, se consiguieron 19 respuestas más, lo que supuso un total de 54 formularios cumplimentados, es decir, un índice de respuesta final del 25,3\%.

\section{Análisis de los datos}

Los datos se introdujeron en una hoja de datos Excel para su análisis. Se calcularon las medias, medianas, desviaciones estándares (DE), valores mínimos y máximos (intervalo) o porcentajes, según los parámetros considerados. Los incrementos de aprendizaje en conocimientos, actitudes y habilidades se calcularon como la diferencia de puntuación entre después y antes de la realización del curso. Para la representación gráfica de las puntuaciones se emplearon diagramas de caja. Cada caja representa la distribución del $50 \%$ de los datos con percentil del $75 \%$ (parte superior de la caja coloreada en gris os-
Tabla IV. Perfil de los asistentes a los cursos de formación.

\begin{tabular}{|c|c|c|c|c|c|}
\hline & & \multicolumn{2}{|c|}{$\begin{array}{l}\text { Total de } \\
\text { asistentes } \\
(n=216)\end{array}$} & \multicolumn{2}{|c|}{$\begin{array}{c}\text { Respuestas al } \\
\text { cuestionario } 2 \\
(n=54)^{\mathrm{a}}\end{array}$} \\
\hline & & $n$ & $\%$ & $n$ & $\%$ \\
\hline \multirow{2}{*}{ Sexo } & Mujeres & 147 & 68,1 & 34 & 63,0 \\
\hline & Hombres & 69 & 31,9 & 20 & 37,0 \\
\hline \multirow{9}{*}{ Formación } & Medicina & 83 & 38,4 & 14 & 25,9 \\
\hline & Farmacia & 46 & 21,3 & 20 & 37,0 \\
\hline & Enfermería & 41 & 19,0 & 9 & 16,7 \\
\hline & Biología & 23 & 10,6 & 2 & 3,7 \\
\hline & Química & 10 & 4,6 & 0 & 0 \\
\hline & Trabajador Social & 4 & 1,8 & 1 & 1,8 \\
\hline & Ciencias & 3 & 1,4 & 1 & 1,8 \\
\hline & Informática & 2 & 0,9 & 1 & 1,8 \\
\hline & Otras $^{b}$ & 4 & 1,8 & 6 & 11,1 \\
\hline \multirow{4}{*}{$\begin{array}{l}\text { Lugar } \\
\text { de trabajo }\end{array}$} & Universidad & 104 & 48,1 & 26 & 48,1 \\
\hline & Hospital & 81 & 37,5 & 14 & 26,0 \\
\hline & Atención primaria & 11 & 5,1 & 1 & 1,8 \\
\hline & Otros ${ }^{c}$ & 20 & 9,3 & 13 & 24,1 \\
\hline
\end{tabular}

a Sólo se dispuso de información sobre la edad de los 54 asistentes que respondieron el cuestionario 2 diferido, siendo la media de 46 años (intervalo: 25-61 años). b Otras especialidades de los asistentes fueron odontología, psicología, derecho y comunicación. ' Como otros lugares de trabajo figuraban laboratorios o centros de investigación, oficinas de farmacia y algunos sin especificar.

curo), mediana (línea media entre las dos tramas grises, línea inferior de la caja gris oscuro o línea superior de la caja gris claro), percentil 25\% (parte inferior de la caja coloreada de gris claro) y sus correspondientes intervalos (extremo superior o inferior representado con un segmento o 'bigote' que sobresale de las cajas).

\section{Resultados}

En la tabla IV se describe el perfil de los asistentes a los cursos de formación y de aquellos que contesta- 
Tabla V. Principales limitaciones para aplicar el ABP en el entorno docente y profesional de los asistentes ${ }^{\mathrm{a}}$.

\begin{tabular}{|c|c|}
\hline & $n$ \\
\hline Excesivo número de alumnos por curso & 12 \\
\hline $\begin{array}{l}\text { Falta de tradición, predisposición } \\
\text { y coordinación con el resto de docentes }\end{array}$ & 11 \\
\hline Inadecuadas infraestructuras & 7 \\
\hline $\begin{array}{l}\text { Tiempo insuficiente para preparar la } \\
\text { nueva metodología en ABP y aplicarla }\end{array}$ & 5 \\
\hline Falta de experiencia y falta de formación & 5 \\
\hline Resistencia del alumnado & 2 \\
\hline Dificultad de evaluación & 2 \\
\hline Necesidad de replantear y redactar nuevos objetivos & 1 \\
\hline \multicolumn{2}{|c|}{$\begin{array}{l}\text { a Se obtuvieron a partir del cuestionario } 2 \text { de opinión enviado por correo } \\
\text { electrónico en respuesta a las preguntas: 'en caso de haber podido aplicar } \\
\text { el ABP, ¿cuál considera que ha sido la principal limitación encontrada?' y } \\
\text { 'en caso de no haber podido aplicar el ABP, ¿cuál ha sido la principal cau- } \\
\text { sa de ello?'. Las } 45 \text { limitaciones se obtuvieron sobre los } 54 \text { asistentes que } \\
\text { respondieron este cuestionario, si bien algunos de ellos anotaron más de } \\
\text { una limitación. }\end{array}$} \\
\hline
\end{tabular}

ron el cuestionario 2 de opinión. Se trataba mayoritariamente de mujeres, provenientes de áreas de la medicina, farmacia, enfermería y biología, y que trabajaban en la universidad o en hospitales. En la figura 1 se reflejan los resultados de satisfacción obtenidos a partir de los 194 cuestionarios impresos (cuestionario 1) cumplimentados por los asistentes al final de cada edición del curso (índice de respuesta del $89,8 \%$ ). La satisfacción con el curso fue muy alta, con una mediana de 4 puntos sobre 5 (media $=4,2$; $\mathrm{DE}=0,7$; intervalo: $2-5$ ). La mediana también fue de 4 al puntuar la documentación entregada (media = 4,3 ; $\mathrm{DE}=0,7$; intervalo: $2-5$ ). Si bien ambas obtuvieron una mediana de 4 , las clases prácticas resultaron levemente mejor puntuadas (media $=4,1 ; \mathrm{DE}=0,9$; intervalo: $1-5$ ) que las teóricas (media $=3,9$; $\mathrm{DE}=$ 0,8; intervalo: $2-5$ ). Tanto los profesores (mediana $=5$; media $=4,4 ; \mathrm{DE}=0,7$; intervalo: $2-5$ ) como la organización (mediana $=5$; media $=4,6$; $\mathrm{DE}=0,6$; intervalo: 2 -5) satisficieron altamente a los asistentes.

El cuestionario impreso también recogía sugerencias o comentarios de los asistentes para la mejora de ediciones posteriores del curso. En las primeras ediciones se solicitaron más clases magistrales y en particular una introducción general sobre la metodología del ABP. Bastantes asistentes a este
Figura 1. Satisfacción con el curso sobre ABP expresada por parte de los asistentes a través del cuestionario 1 . El eje de ordenadas representa los datos de una escala numérica comprendida entre 0 (mínima satisfacción) y 5 (máxima satisfacción) para puntuar los diferentes parámetros del eje de abscisas: valoración general del curso (VAL), documentación entregada (DOC), contenido de las clases teóricas (TEO), adecuación de los ejercicios prácticos (EJE), contribución de los dos profesores del curso (PRO) y aportación de Fundación Dr. Antonio Esteve como institución organizadora (ORG).

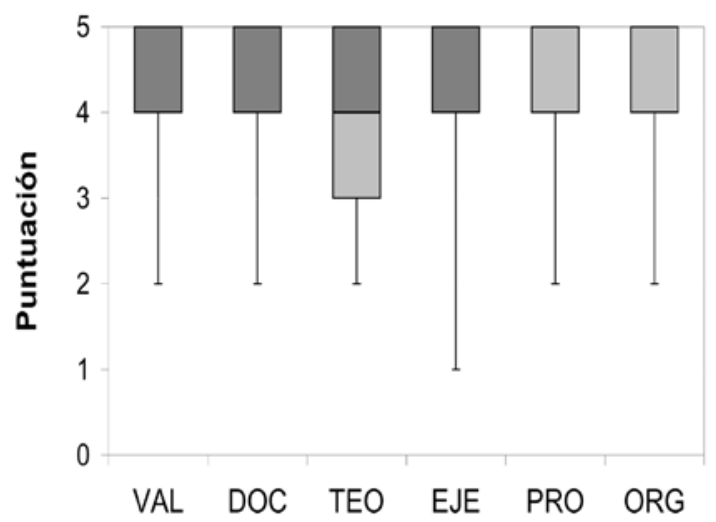

primer curso general sobre las bases del ABP mostraron su interés por dar continuidad a su formación con otros cursos que completaran aspectos adicionales del ABP, como la preparación de casos y cómo actuar de tutor-facilitador.

Las 54 personas que respondieron el cuestionario 2 de opinión tenían una media de edad de 46 años (intervalo: 25-61 años), mayoritariamente mujeres (63,0\%) (Tabla IV). De los 54 participantes, tan sólo 10 (18,5\%) manifestaron haber participado en alguna actividad relacionada con el ABP antes de asistir al curso. Y, en realidad, sólo cinco de ellos (9,3\%) habían impartido algún curso mediante el ABP.

En la figura 2 se representa el aprendizaje adquirido con el curso según la opinión de los asistentes. En el bloque de conocimientos, los parámetros evaluados en su conjunto reflejaron un incremento del aprendizaje medio de 2,6 puntos sobre un máximo de $5(\mathrm{DE}=1,1$; intervalo: $0-5)$. Se pasó de una mediana de conocimientos de cero antes de realizar el curso (media $=0,8 ; \mathrm{DE}=1,0$; intervalo: $0-3$ ) a una mediana de 3 al finalizarlo (media $=3,4 ; \mathrm{DE}=1,0$; intervalo: 1-5), es decir, un incremento de 3 puntos. En particular, fueron los conocimientos respecto al $\mathrm{ABP}$ en grupos pequeños los que alcanzaron un aprendizaje superior, con un incremento medio de 
Figura 2. Aprendizaje experimentado con la realización del curso según la opinión de los asistentes mediante el cuestionario 2. El eje de ordenadas representa los datos de una escala numérica que refleja el aprendizaje en conocimientos, actitudes y habilidades calculados a partir de la diferencia de puntuación entre después y antes de la realización del curso. Las puntuaciones de antes y después del curso se basaban en una escala de 0 (mínima puntuación) a 5 (máxima puntuación). En el eje de abscisas figuran los diferentes parámetros evaluados (véanse las preguntas específicas en la tabla III) respecto a los conocimientos: fundamentos del $A B P$ en general (GEN), $A B P$ en grupos pequeños (GPQ), $A B P$ en grupos grandes (GGR), evaluación (EVA) e implementación del ABP (IMP); respecto a las actitudes: necesidad del ABP (NEC), dificultades (DIF) y puesta en marcha de iniciativas (INI); respecto a las habilidades: preparación de objetivos (OBJ), actuar como tutor (TUT) y preparación de un caso (CAS).

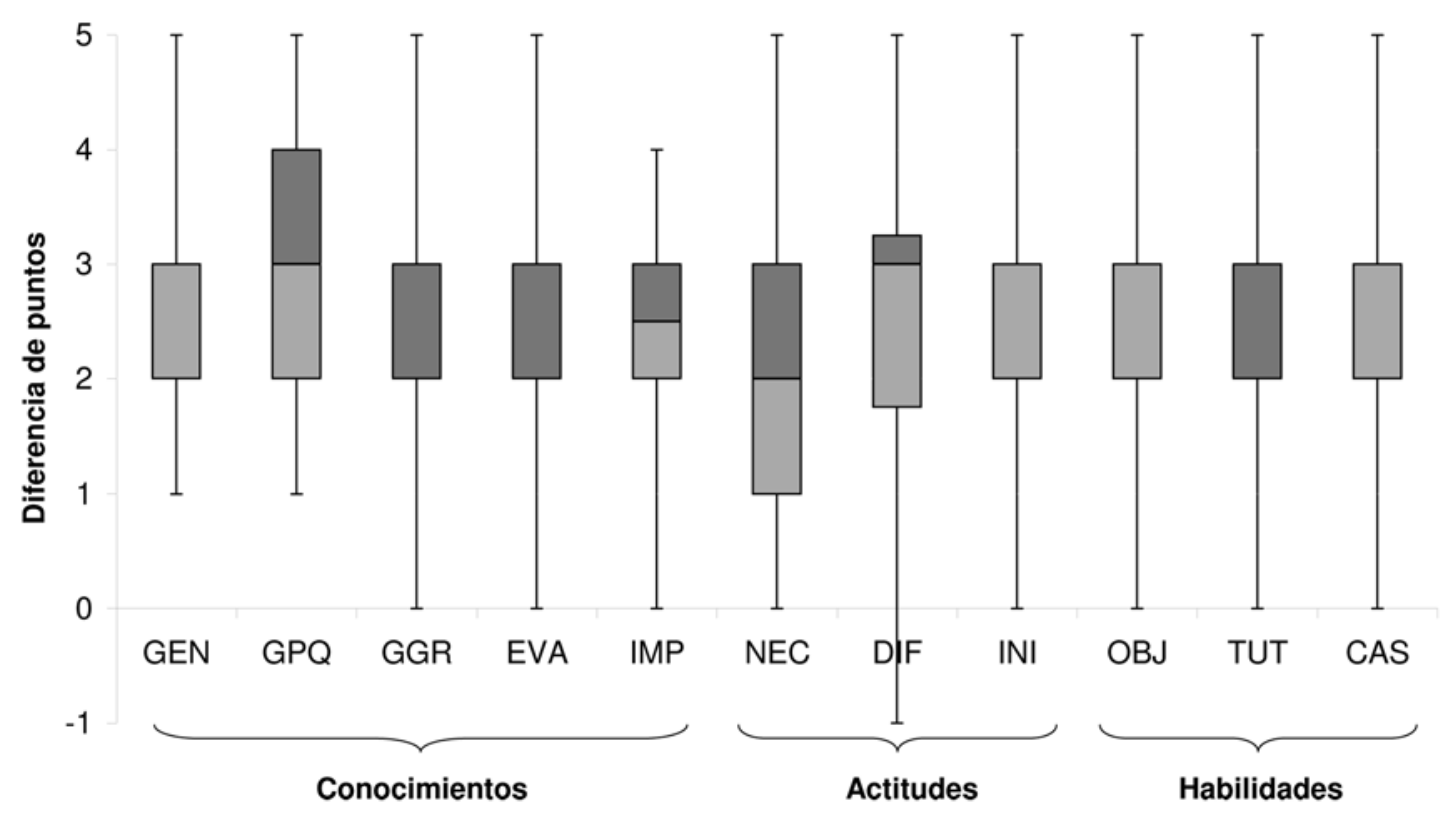

2,9 puntos $(\mathrm{DE}=1,1$; mediana $=3)$ y donde todos los asistentes manifestaron algún tipo de mejora entre antes y después del curso (intervalo: 1-5). En cuanto a las actitudes en su conjunto, los asistentes manifestaron que con la realización del curso experimentaron un incremento medio de 2,4 puntos $(\mathrm{DE}=1,5$; intervalo: -1 a 5 ; mediana $=3)$. Se pasó de una mediana de actitud de 1 antes de realizar el curso (media $=1,6 ; \mathrm{DE}=1,4$; intervalo: $0-5$ ) a una mediana de 4 al finalizarlo (media $=3,9 ; \mathrm{DE}=0,9$; intervalo: 1-5), es decir, también un incremento de 3 puntos. En particular, se observó un menor incremento en la actitud frente a la necesidad de emplear el $\mathrm{ABP}$ en docencia, con una media de 2,3 $(\mathrm{DE}=$ 1,4; intervalo: 0-5; mediana $=2$ ). Cabría comentar la puntuación por parte de dos asistentes que tras la realización del curso todavía fueron más conscientes de las dificultades para aplicar el ABP, por lo que su actitud tras el curso decreció y quedó reflejada con valor negativo (intervalo inferior del parámetro DIF de actitudes en la figura 2). Sin embargo, la actitud por intentar emprender iniciativas con el ABP creció 2,4 puntos de media con la realización del curso $(\mathrm{DE}=1,4$; intervalo: 0-5; mediana $=3$ ). En lo que respecta a las habilidades adquiridas con el curso, en conjunto incrementaron 2,5 puntos de media $(D E=1,2$; intervalo: $0-5$; mediana $=3$ ), siendo el aumento ligeramente menor en el caso específico de las habilidades de actuar como tutor (media $=2,4 ; D E=1,2$, intervalo: $0-5$; mediana $=2$ ).

Los asistentes también opinaron sobre la posibilidad de aplicar lo aprendido tras acudir a los cursos de formación. El 38,9\% de ellos $(n=21)$ manifestó haber aplicado la metodología docente del ABP tras la realización del curso. En una segunda pregunta, la mayoría $(n=36 ; 66,7 \%)$ declaró haber podido aplicar en su docencia habitual algún aspecto aprendido durante el curso. La tabla V enumera las principales limitaciones que, según los participantes en la encuesta, se habían encontrado en el momento de aplicar el ABP en su entorno, o bien aquellas que justificaban el no haberlo podido aplicar. El excesivo número de estudiantes, los problemas de un entorno adverso por parte de los centros docentes y la 
resistencia de los propios profesionales parecen ser las principales barreras a superar para la adecuada implementación del ABP. Nueve encuestados (16,7\%) manifestaron no haber podido aplicar la metodología del ABP en sus programas docentes por no haber tenido todavía actividad académica desde la realización del curso.

En otro apartado del formulario se interrogó a los encuestados sobre si consideraban necesario impartir cursos de formación en ABP por parte de los centros docentes ubicados en hospitales, facultades y universidades. Se obtuvo una puntuación media de 4,3 sobre 5 apoyando esta opinión ( $D E=1,1$; intervalo: 0-5; mediana $=5$ ).

Finalmente, si bien ya se había medido mediante el cuestionario 1, en el último apartado del cuestionario 2 se volvió a preguntar sobre la satisfacción con el curso de ABP, ahora con cierta perspectiva temporal. Medida mediante la pregunta ‘ ¿recomendaría positivamente este seminario a un/a amigo/a?', se obtuvo una puntuación media de 4,4 puntos $(\mathrm{DE}=$ 1,0; intervalo: 0-5; mediana $=5$ ).

\section{Discusión}

El análisis de estas encuestas realizadas tras 11 ediciones del curso, en las cuales han participado más de 200 docentes, permite afirmar que tanto el formato como los contenidos del seminario de formación fueron bien acogidos y que, según la opinión de los asistentes, poder asistir al curso supuso una mejora en sus competencias. Tras el curso, a pesar de las dificultades de implementación de esta estrategia docente, un porcentaje considerable de participantes manifestaron haber aplicado sus conocimientos sobre el ABP en su práctica profesional. Estos primeros datos del trabajo son suficientes para que profesores y organizadores estén satisfechos con el esfuerzo que ha supuesto la puesta en marcha de las ediciones del curso de forma itinerante en diferentes ciudades españolas. En general, iniciativas formativas como ésta suelen tener buena acogida y contribuir en la mejora de las competencias [11].

La contribución del ABP, como método docente, en mejorar el aprendizaje de los estudiantes está demostrada [12-15]. Además, teniendo en cuenta que nuestro país está inmerso en el entorno del Espacio Europeo de Educación Superior y el planteamiento de la Declaración de Bolonia, la implementación de innovaciones como el ABP debería considerarse indispensable en nuestras universidades. Sin embargo, no parece muy atrevido afirmar que actualmente, en nuestro entorno, la implementación del ABP en docencia es todavía escasa y las iniciativas al respecto son puntuales. Ello no pretende restar mérito alguno a otras experiencias previas [1622] ni a los diferentes programas de formación implementados por la mayoría de universidades que, sin embargo, no siempre han evaluado su impacto tan extensivamente como el presente estudio. Probablemente, la acogida de este curso reforzaría de forma indirecta la anterior afirmación de una escasa implementación del ABP en España, puesto que son muchas las instituciones y los docentes que han mostrado su interés en impartir este tipo de formación. Los propios encuestados también reconocieron la necesidad de estos cursos y recomendaban asistir a posteriores ediciones. Entre los asistentes a este primer curso general sobre el ABP se observó el interés por dar continuidad a su formación con otros cursos que completaran aspectos adicionales del ABP, como la preparación de casos o cómo actuar de tutor-facilitador. Sin embargo, llama algo la atención que, a través de nuestra encuesta, no se reclamara otro tipo de cursos más avanzados sobre ABP o cursos de mantenimiento para docentes, tal como se han solicitado en otros estudios $[11,23]$.

Otros cursos de formación avalan la tradición docente de la Fundación Dr. Antonio Esteve [8,24], si bien la experiencia que se recoge en este artículo es la primera desarrollada específicamente sobre metodología docente. Estos cursos sobre ABP han facilitado posteriormente la organización de un nuevo seminario de formación para tutores-facilitadores en ABP y de un curso de ABP en línea en colaboración con la Universitat de Girona [25]. Siguiendo la idea de que lo que no se publica no existe, la mayoría de iniciativas de la Fundación Dr. Antonio Esteve se describen en uno $u$ otro formato de publicación [26]. Es importante primero evaluar y después publicar esta información para dar a conocer las diferentes iniciativas existentes a fin de que puedan servir como referencia para otros docentes e instituciones.

A través de las bases bibliográficas consultadas, no se han encontrado publicaciones específicas sobre cursos itinerantes orientados a la formación de los docentes, como los cursos que se presentan en este artículo. Por otro lado, sí se han hallado múltiples experiencias formativas en el extranjero llevadas a cabo sobre el ABP, tanto de cursos de iniciación como talleres de formación para actuar como tutores [1,23,27-30]. También se deben destacar tres publicaciones que presentan la experiencia del ABP en el área de odontología [31-33]. Estos trabajos describen unos cursos de formación orientados a tutores-facilitadores, en los que participaron más de un centenar de futuros docentes. Se detallan las carac- 
terísticas de talleres que se impartieron durante tres años en diferentes ediciones y se analizan los datos de una encuesta posterior. Otro trabajo ya citado [11], siguiendo una evaluación antes y después de un curso de tres días para formar a tutores de ABP, evidencia un incremento significativo de los conocimientos sobre el ABP por parte de los asistentes gracias a la impartición del curso. Finalmente, otros trabajos describen experiencias de países en los que se están implementando progresivamente nuevos métodos de docencia y se han organizado talleres de formación sobre ABP con resultados muy satisfactorios [34-36]. También se ha publicado alguna experiencia formativa tras varios años de utilización de videos para la formación de tutoresfacilitadores [37].

El perfil de los asistentes al seminario de formación se relacionaba directamente con el ámbito de trabajo de la Fundación Dr. Antonio Esteve, es decir, se trataba de profesionales procedentes de áreas de ciencias de la salud que trabajan en centros académicos o asistenciales. La mayoría relataba poca o ninguna experiencia en el ABP y prácticamente para todos suponía su primer curso de formación en ABP. Tan sólo 10 personas manifestaron haber participado en alguna actividad relacionada con el ABP antes de asistir al curso. Y, en realidad, sólo la mitad de ellas habían impartido cursos con la metodología del ABP. Ello constata la necesidad de estos cursos y el interés de los docentes por formarse en nuevas estrategias docentes.

Como también se realizó en otros cursos sobre el ABP [32], en el que aquí se describe se utilizó la propia estrategia de ABP para impartirlo. Dado que a través de los resultados de los cuestionarios de satisfacción de la primera edición se solicitó una introducción general sobre el ABP, posteriormente se incorporó una clase magistral introductoria en las siguientes ediciones. La combinación del ABP con las clases magistrales conllevó que los asistentes asumieran el rol de estudiantes, similar a un roleplay. Si bien, a menudo, ello podía generar cierta sorpresa entre los participantes, basado en la experiencia de dos de los autores de este artículo se consideró que era ésta la mejor manera de que los futuros tutores en ABP sintieran directamente la percepción de los estudiantes noveles en esta metodología. Por circunstancias organizativas, los grupos fueron más numerosos de lo recomendado [38]. Por ello, los talleres de ABP se realizaron tanto en grupos pequeños como grandes $y$, por este motivo, se impartieron las bases de ambos formatos.

En general, se considera que los principales problemas que limitan la implementación del ABP in- cumben a aquellos derivados del entorno docente, de los profesores, de los alumnos o de los propios grupos de ABP, además de la inadecuación o falta de casos o escenarios [39]. En nuestro estudio, un grupo considerable de encuestados no pudo enumerar los motivos que, según su punto de vista, limitaban la implementación del ABP en su entorno. Ello se debía a que no habían tenido oportunidad de impartir todavía docencia desde la finalización del seminario de formación. Quienes respondieron a la pregunta, opinaron mayoritariamente que el número excesivo de estudiantes por clase suponía el principal problema. Si bien ello podría solventarse con el ABP para grupos grandes, de la encuesta se deduce que los asistentes al curso prefieren iniciarse con el ABP para grupos pequeños, aplicándolo en determinadas asignaturas optativas o en cursos de posgrado y doctorado, en los cuales el número de asistentes es menor. Por otro lado, sorprende a través de la encuesta no haber recogido más problemas sobre la implementación del ABP, como la necesidad de disponer de casos válidos, tal como destacaron otros autores [39]. Es realmente preocupante que las mismas instituciones o el propio entorno docente, por el motivo que fuere, constituyan todavía un impedimento para la adecuada implementación del ABP en las universidades españolas. De nuevo se cae en el error de no exigir a los programas tradicionales ya establecidos, y sin comprobada evidencia de efectividad, que demuestren lo que sí se exige a los que introducen cambios como el ABP [40].

Este trabajo presenta la limitación del reducido índice de respuesta obtenido en concreto con el segundo cuestionario. Consecuentemente, ello imposibilita la realización de análisis por estratos, como la evaluación de diferencias según edad, sexo, formación o procedencia de los asistentes. Por otro lado, debe tenerse en cuenta la subjetividad en la cumplimentación de la encuesta diferida. En ella se preguntaba a los participantes sobre su percepción de las competencias antes y después del curso, sin realizarse una evaluación objetiva del estado de dichas competencias. Además, una limitación propia del curso se fundamentaba en su propio contenido. Se trataba de cursos de iniciación al ABP que, en dos días, no podían profundizar en aspectos también muy importantes como la adquisición de habilidades para actuar como tutor-facilitador ni incluir ejercicios específicos sobre cómo preparar el texto de los casos, tal como algunos participantes reclamaron. Como en su momento ocurrió en otras experiencias [28], este tipo de formación en ABP es básica y debe servir como punto de partida para 
posteriormente incidir en aspectos de formación mucho más específicos. También cabría comentar que para algunos participantes de la encuesta, desde que asistieron al curso hasta la cumplimentación del cuestionario 2 sólo habían transcurrido cuatro meses. Por tanto, en estos casos no hubo tiempo suficiente para analizar las posibilidades de aplicación del curso. En el futuro, y como continuidad de este estudio, podría ser útil reevaluar la aplicación práctica de estos seminarios de formación a más largo plazo y analizar si los asistentes pudieron implementar lo aprendido en su medio.

En conclusión, parece lógico insistir en la necesidad de optar cada vez más por el aprendizaje autodirigido en la docencia, donde el ABP ejerce un papel clave. Además, el ABP ofrece la oportunidad de que los estudiantes desarrollen habilidades y valores profesionales, como son las habilidades de aprendizaje, de comunicación, el trabajo en equipo y las relaciones interpersonales. Estos aspectos se presentaron y discutieron en los cursos que describe este artículo, incluyendo su evaluación. Es por ello que los resultados de este estudio evidencian la necesidad, la importancia y la utilidad de los cursos de formación, en este caso itinerantes, sobre el ABP dirigidos a los docentes. Creemos que la experiencia puede aplicarse a todas las instituciones con intención de implementar el ABP en su docencia, si bien el formato y contenidos del curso deberían adaptarse a las diferentes necesidades de los centros. En definitiva, el objetivo final de los cursos debería ser brindar más herramientas a los docentes para poder, en consecuencia, mejorar la formación de los estudiantes.

\section{Bibliografía}

1. Baños JE, Pérez J. Cómo fomentar las competencias transversales en los estudios de ciencias de la salud: una propuesta de actividades. Educ Med 2005; 8: 216-25.

2. Branda LA. El aprendizaje basado en problemas: el resplandor tan brillante de otros tiempos. In Araújo UF, Sastre-Vilarrasa G, eds. El aprendizaje basado en problemas. Una nueva perspectiva de la enseñanza en la universidad. Barcelona: Gedisa; 2008. p. 17-46.

3. Cònsul-Giribet $M$, ed. Historia de un cambio. Un currículo integrado con el aprendizaje basado en problemas. Barcelona: Enciclopèdia Catalana; 2007.

4. Bernabeu-Tamayo MD. Innovación curricular en la Escola Universitària d'Infermeria Vall d'Hebron de Barcelona: diseño e implementación del ABP. In Araújo UF, SastreVilarrasa G, eds. El aprendizaje basado en problemas. Una nueva perspectiva de la enseñanza en la universidad. Barcelona: Gedisa; 2008. p. 191-205.

5. Bosch F, Baños JE. Tendiendo puentes: la utilidad de la historia de la ciencia para comprender el proceso de investigación y desarrollo de medicamentos. Educ Med 2010; 13: 255-62.

6. Branda LA. El aprendizaje basado en problemas. De herejía artificial a res popularis. Educ Med 2009; 12: 11-23.
7. Hernández-Abenza L, Hernández-Torres C. Hacia un modelo dinámico y eficiente de formación de profesorado. REIFOP 2011; 14: 53-66.

8. Bosch F, Baños JE, Erill S. Medical education beyond patient-physician relationship: an experience from the Esteve Foundation. Educ Med 2004; 7: 47-8.

9. Fundación Dr. Antonio Esteve. URL: www.esteve.org. [21.09.2012].

10. Cantillon P, Hutchinson L, Wood D, eds. Aprendizaje y docencia en medicina. Cuadernos de la Fundación Dr. Antonio Esteve, n. ${ }^{\circ}$ 5. Barcelona: Prous Science; 2006.

11. Baral N, Paudel BH, Das BKL, Aryal M, Gautam A, Lamsal M. Preparing tutors for problem-based learning: an experience from B. P. Koirala Institute of Health Sciences, Nepal. Kathmandu University Medical Journal 2010; 8: 141-5.

12. Davis MH, Harden RM. Problem based learning: a practical guide. AMEE Medical Education Guide no. 15. Med Teach 1999; 21: 130-40.

13. Koh GC, Khoo HE, Wong ML, Koh D. The effects of problembased learning during medical school on physician competency: a systematic review. CMAJ 2008; 178: 34-41.

14. Polyzois I, Claffey N, Mattheos N. Problem-based learning in academic health education. A systematic literature review. Eur J Dent Educ 2010; 14: 55-64.

15. Schmidt H. A review of the evidence: effects of problembased learning in students and graduates of Maastricht Medical School. In Van Berkel HJM, Scherpbier A, Hillen H, Van der Vleuten C, eds. Lessons from problem-based learning. Oxford: Oxford University Press; 2010. p. 227-47.

16. Baños JE. El aprendizaje basado en problemas en los planes de estudio tradicionales: ¿una alternativa posible? Educ Med 2001; 4: 4-12

17. Quintanilla M, Bernaus E, Guillamet A, Fernández A. Learning to solve problems in clinical practice. Educ Med 2004; 7: 104.

18. Alfonso-Roca MT, Sáez-Méndez L, Serrano-Casarrubio P. Changing Spanish medical studies: our experience. Educ Med 2004; 7: 268.

19. Cònsul-Giribet M. La formación del profesorado; un cambio de cultura docente. In Cònsul-Giribet M, ed. Historia de un cambio. Un currículo integrado con el aprendizaje basado en problemas. Barcelona: Enciclopèdia Catalana; 2007. p. 71-8.

20. Branda LA, ed. L'aprenentatge basat en problemes. Bellaterra: Universitat Autònoma de Barcelona; 2009.

21. Alcolea-Cosín MT, Oter-Quintana C, Martínez-Ortega RM, Sebastián-Viana T, Pedraz-Marcos A. Aprendizaje basado en problemas en la formación de estudiantes de enfermería. Impacto en la práctica clínica. Educ Med 2012; 15: 23-30.

22. Castro-Sánchez AM, Aguilar-Ferrándiz ME, Matarán-

Peñarrocha GA, Iglesias-Alonso A, Fernández-Fernández MJ, Moreno-Lorenzo C. Problem based learning approaches to the technology education of physical therapy students. Med Teach 2012; 34: e29-45.

23. McLean M. What can we learn from facilitator and student perceptions of facilitation skills and roles in the first year of a problem-based learning curriculum? BMC Med Educ 2003; 3: 9.

24. Bosch F, Serés E. Demanda de formación en profesionales sanitarios. Educ Med 2010; 13(3): 145-7.

25. Carrión C, Aymerich M, Castells X, Xifro X, Soler M, Branda LA. Disseny d'un curs on-line sobre l'aprenentatge basat en problemes (ABP). Presentación oral al III Congreso Europeo de Tecnologías de la Información en la Educación y en la Sociedad: una visión crítica. Libro de Resúmenes 2012. p. 310-1.

26. Fundación Dr. Antonio Esteve (publicaciones). URL: http:// esteve.org/aw/Home/Secciones_Web/ e/Publicacions/. [19.09.2012].

27. Branda LA, Sciarra AF. Faculty development for problembased learning. Annals of Community-Oriented Education 1995; 8: 195-208.

28. Wetzel MS. Developing the role of the tutor/facilitator. Postgrad Med J 1996; 72: 474-7.

29. Bollela VR, Gabarra MH, Da Costa C, Lima RC. Students and tutors' social representations of assessment in problem- 
based learning tutorials supporting change. BMC Med Educ 2009; 9: 30.

30. Hendry GH. Problem-based learning tutors' conceptions of their development as tutors. Med Teach 2009; 31: 145-50

31. Dalrymple KR, Wuenschell CW, Rosenblum A, Paine M, Crowe D, Von Bergmann HC, et al. PBL core skills faculty development workshop 1: an experiential exercise with the PBL process. J Dent Educ 2007; 71: 249-59.

32. Wuenschell CW, Dalrymple KR, Shuler CF. PBL core skills faculty development workshop 2: training faculty in group learning facilitation skills through role-modeling and roleplay activities. J Dent Educ 2007; 71: 606-18.

33. Dalrymple KR, Wong S, Rosenblum A, Wuenschell CW, Paine M, Schuler CF. PBL core skills faculty development workshop 3: understanding PBL process assessment and feedback via scenario-based discussions, observation, and role-play. J Dent Educ 2007; 71: 1561-73.

34. Jafri W, Mumtaz K, Burdick WP, Morahan PS, Freeman R, Zehra T. Improving the teaching skills of residents as tutors/ facilitators and addressing the shortage of faculty facilitators for PBL modules. BMC Med Educ 2007; 7: 34.
35. Sadaf S, Ali SK, Zuberi RW. Problem-based learning: enhancing tutors' facilitation skills using structured small group experiential learning. Educ Health (Abingdon) 2009; $22: 96$. 36. Saeed SA, Zia N, Qazi Y. Facilitation skills of basic sciences faculty and fresh medical graduates: an AKU experience. Procedia - Social and Behavioral Sciences 2010; 2: 1316-20. 37. Bosse HM, Huwendiek S, Skelin S, Kirschfink M, Nikendei C. Interactive film scenes for tutor training in problem-based learning (PBL): dealing with difficult situations. BMC Med Educ 2010; 10: 52.

38. Branda LA. El traje hecho a la medida, (re)corte y confección [Internet]. URL: http://www.udg.edu/fm/UnitatdEducacio Medica/InformacioiPublicacions/tabid/18032/language/ ca-ES/Default.aspx.

39. Dolmans DH, De Grave W, Wolfhagen IH, Van der Vleuten CP. Problem-based learning: future challenges for educational practice and research. Med Educ 2005; 39: 732-41.

40. Branda LA. Reflexiones sobre las cosas pasadas. In CònsulGiribet M, ed. Historia de un cambio. Un currículo integrado con el aprendizaje basado en problemas. Barcelona: Enciclopèdia Catalana; 2007. p. 11-31. 